\title{
On addition-subtraction chains of numbers with low Hamming weight
}

\author{
Dustin Moody ${ }^{1}$ and Amadou Tall ${ }^{2}$ \\ ${ }^{1}$ Computer Security Division, National Institute of Standards and Technology \\ Gaithersburg, Maryland, 20899, USA \\ e-mail: dustin.moody@nist.gov \\ ${ }^{2}$ Departement de Mathematiques et Informatique, Université Cheikh Anta Diop de Dakar \\ Dakar, Senegal \\ e-mail: amadou7.tall@ucad.edu.sn
}

Received: 5 September 2018

Accepted: 31 May 2019

\begin{abstract}
An addition chain is a sequence of integers such that every element in the sequence is the sum of two previous elements. They have been much studied, and generalized to additionsubtraction chains, Lucas chains, and Lucas addition-subtraction chains. These various chains have been useful in finding efficient exponentiation algorithms in groups. As a consequence, finding chains of minimal length is critical. The main objective of this paper is to extend results known for addition chains to addition-subtraction chains with Lucas addition-subraction as a tool to construct such minimal chains. Specifically, if we let $\ell^{-}(n)$ stand for the minimal length of all the Lucas addition-subtraction chains for $n$, we prove $\left|\ell^{-}(2 n)-\ell^{-}(n)\right| \leq 1$ for all integers $n$ of Hamming weight $\leq 4$. Thus, to find a minimal addition-subtraction chains for low Hamming weight integers, it suffices to only consider odd integers.
\end{abstract}

Keywords: Addition chains, Subtraction chains, Addition-subtraction chains, Lucas chains.

2010 Mathematics Subject Classification: 11Y55, 11 Y16.

\section{Introduction}

Addition chains are an important tool to perform fast exponentiation in groups $[4,5,7,10,11$, $12,16]$. In particular, in groups where computing $-P$ is as easy as computing $P$ (where $P$ is an element of the group), then addition-subtraction chains become more interesting than the general 
addition chains $[1,3,7,8,10,11,12]$. We begin by defining the various types of addition chains and their generalizations. Let $n$ be an integer.

\subsection{Definitions}

Definition 1 ([6]). A sequence of positive integers $C=\left\{1=a_{0}, a_{1}, \cdots, a_{l}=n\right\}$ is called an addition chain for $n$ if and only if for every $a_{i} \in C$ (with $i>0$ ), there exists $a_{j}, a_{k} \in C$ with $j, k<i$ such that

$$
a_{i}=a_{j}+a_{k} .
$$

As an example, the following is an addition chain for 42:

$$
\{1,2,4,5,10,11,21,42\} \text {. }
$$

Definition 2 ([14]). A sequence of positive integers $C=\left\{1=a_{0}, a_{1}, \ldots, a_{l}=n\right\}$ is called an addition-subtraction chain for $n$ if and only if for every $a_{i} \in C$, there exists $a_{j}, a_{k} \in C$ with $j, k<i$ such that

$$
a_{i}=a_{j}+a_{k} \text { or } a_{i}=a_{j}-a_{k} .
$$

We now give an addition-subtraction chain for 42:

$$
\{1,2,3,4,8,11,22,44,42\} \text {. }
$$

Definition 3 ([9]). A sequence of positive integers $C=\left\{1=a_{0}, a_{1}, \ldots, a_{l}=n\right\}$ is called a Lucas addition chain if and only if for every $a_{i} \in C($ with $i>0)$, there exists $a_{j}, a_{k} \in C$ with $j, k<i$ such that

$$
a_{i}=a_{j}+a_{k} \text { where either } a_{j}=a_{k} \text { or }\left|a_{j}-a_{k}\right| \in C
$$

The addition chain we gave above for 42 is not a Lucas addition chain, since $5=4+1$, but $4-1$ is not in the chain. An example of a Lucas addition chain for 42 is

$$
\{1,2,3,4,7,14,28,42\} \text {. }
$$

Definition 4 ([13]). A sequence of positive integers $C=\left\{1=a_{0}, a_{1}, \ldots, a_{l}=n\right\}$ is called a Lucas addition-subtraction chain if and only iffor any $a_{i} \in C$ (with $\left.i>0\right)$, there exists $a_{j}, a_{k} \in C$ with $j, k<i$ such that

$$
a_{i}= \begin{cases}a_{j}+a_{k} & \text { and }\left|a_{j}-a_{k}\right| \in C \cup\{0\}, \\ & \text { or } \\ a_{j}+1, & \\ & \text { or } \\ a_{j}-a_{k} . & \end{cases}
$$


The addition-subtraction chain we gave above is not a Lucas addition-subtraction chain for 42 , because $11=3+8$, but $8-3$ is not in the chain. An example of a Lucas addition-subtraction chain is given by

$$
\{1,2,3,6,12,24,48,42\} \text {. }
$$

In this work, we will focus on Lucas addition-subtraction chains [13]. These chains have a weaker restriction than Lucas addition chains. This allows these chains to have shorter chains than Lucas addition chains for infinitely many integers. Lucas addition-subtraction chains are one of the simpler known methods for computing addition-subtraction chains $[15,13]$. It is therefore important to see what properties of addition chains (and Lucas addition chains and additionsubtraction chains) are true for the Lucas addition-subtraction chains.

Formally, the length of a chain is the number of elements in the chain, except we do not count $a_{0}=1$. That is, the length of the chain $\left\{a_{0}=1, a_{1}, \ldots, a_{l}\right\}$ is $l$. We are usually interested in chains with as few elements as possible. Let $\ell(n)$ and $\ell^{-}(n)$ denote the minimal lengths of an addition chain and addition-subtraction chain for $n$ respectively. Similarly, we define $\ell_{L}(n)$ and $\ell_{L}^{-}(n)$ for the Lucas version of the same type of chains.

Recall that the Hamming weight $h(n)$ of a positive integer $n$ is the number of 1 's in the binary expansion of $n$. Concretely, if $n=\sum e_{i} 2^{i}$, with $e_{i} \in\{0,1\}$, then the Hamming weight of $n$ is $h(n)=\sum e_{i}$. We also recall the definition of the non-adjacent form of any integer $n$, which gives a signed representation of $n$ with minimal weight. The non-adjacent form of $n$ is the unique signed-digit representation $n=\sum k_{i} 2^{i}$, with $k_{i} \in\{-1,0,1\}$ and $k_{i} k_{i+1}=0$. We define $\bar{s}_{2}(n)=\sum\left|k_{i}\right|$ as the weight of the non-adjacent form of $n$.

\subsection{Motivation}

We note that because an addition chain is also an addition-subtraction chain, then $\ell^{-}(n) \leq \ell(n)$ for any $n$. We similarly have that

$$
\ell^{-}(n) \leq \ell_{L}^{-}(n) \leq \ell_{L}(n), \quad \ell(n) \leq \ell_{L}(n),
$$

for any $n$. However, the relationship between the values of $\ell(n)$ and $\ell_{L}^{-}(n)$ is not so simple. For example, there are infinitely many integers such that $\ell_{L}^{-}(n) \leq \ell(n)$ but at the same time, we have infinitely many integers such that $\ell_{L}^{-}(n) \geq \ell(n)$ (see $[13,14]$ ).

This paper investigates the minimal length of Lucas addition-subtraction chains for integers with low Hamming weight $h(n)$. Our main result will be to prove that if $h(n) \leq 4$, then

$$
\ell_{L}^{-}(n)+1=\ell_{L}^{-}(2 n) .
$$

Equation (1) is known to hold for addition chains [6], as well as for addition-subtraction chains (when $h(n) \leq 4$ ) [14]. We thus show that in order to find the minimal length of a Lucas additionsubtraction chain, it suffices to only consider odd integers (at least for integers with $h(n) \leq 4$ ). As a consequence, we establish that Lucas addition-subtraction chains give minimal additionsubtraction chains for integers $n$, with $h(n) \leq 4$. Our proof will also be constructive, providing 
a method to construct these minimal Lucas addition-subtraction chains (and addition-subtraction chains), for all integers of Hamming weight $\leq 4$.

For several of our proofs, we will need an important theorem due to Volger [14]. For integers of low Hamming weight, Volger's results give the length of a minimal addition-subtraction chain.

Theorem 1.1 (Volger [14]). Let $\ell^{-}(n)$ be the minimal length of all addition-subtraction chains for $n$, then:

$$
\begin{aligned}
& \text { 1. } \ell^{-}\left(2^{a}\right)=a, \\
& \text { 2. } \ell^{-}\left(2^{a}+2^{b}\right)=a+1, \quad \text { for all } a>b, \\
& \text { 3. } \ell^{-}\left(2^{a}-2^{b}\right)=a+1, \quad \text { for all } b \leq a-3, \\
& \text { 4. } \ell^{-}\left(2^{a}+2^{b}+2^{c}\right)=a+2, \quad \text { for all } a, b, c \text { such that } c<b \leq a-2, \\
& \text { 5. } \ell^{-}\left(2^{a}+2^{a-1}+2^{b}\right)=a+2, \quad \text { for all } b \leq a-4, \\
& \text { 6. } \ell^{-}(n) \geq\lfloor\log (n)\rfloor+2, \quad \text { if } \bar{s}_{2}(n) \geq 3 .
\end{aligned}
$$

We note there are two cases for integers of Hamming weight 3 not covered by Volger. The first is when $n=2^{a}+2^{a-1}+2^{a-2}$. However, in this case we observe $n=2^{a+1}-2^{a-2}$, and so by Volger's third result, $\ell^{-}(n)=a+2$. The remaining case is when $n=2^{a}+2^{a-1}+2^{a-3}$. The non-adjacent form is $n=2^{a+1}-2^{a-1}+2^{a-3}$, and so by part 6 of Volger's results we know $\ell^{-}(n) \geq a+2$ and since it is easy to construct an addition-subtraction chain for $n$ of length $a+2$, then $\ell^{-}(n)=a+2$. Combining these two cases with 4) and 5) above, we've shown $\ell^{-}(n)=a+2$, for any $n=2^{a}+2^{b}+2^{c}$, where $a>b>c$.

\section{Minimal lengths for low Hamming weight numbers}

We will start by quantifying $\ell_{L}^{-}(n)$ for all integers of Hamming weight less than four. Similar results are known for addition chains and addition-subtraction chains [6, 14]. The minimal lengths we prove for Lucas addition-subtraction chains are equal to the minimal lengths for additionsubtraction chains (for integers with $h(n) \leq 4$ ).

Theorem 2.1. Let $n$ be a positive integer. Depending on the Hamming weight of $n$, we have the following:

- For $h(n)=1, n=2^{a}$, we have $\ell_{L}^{-}(n)=a$.

- $\operatorname{For} h(n)=2, n=2^{a}+2^{b}$, we have $\ell_{L}^{-}(n)=a+1$.

- For $h(n)=3, n=2^{a}+2^{b}+2^{c}$, we have $\ell_{L}^{-}(n)=a+2$.

- For $h(n)=4, n=2^{a}+2^{b}+2^{c}+2^{d}$, we have $\ell_{L}^{-}(n)=a+2$ or $a+3$.

The proof will be given by the next three propositions. The first covers integers which are either a power of 2 , or a sum or difference of powers of 2 . 
Proposition 2.1.1. Let $n$ be a positive integer.

1. If $n=2^{a}$, then $\ell_{L}^{-}(n)=a$.

2. If $n=2^{a}-2^{a-1}$, then $\ell_{L}^{-}(n)=a-1$.

3. If $n=2^{a}-2^{a-2}$, then $\ell_{L}^{-}(n)=a$.

4. If $n=2^{a}-2^{b}$ with $b \leq a-3$, then $\ell_{L}^{-}(n)=a+1$.

5. If $n=2^{a}+2^{b}$ with $b<a$, then $\ell_{L}^{-}(n)=a+1$.

Proof. 1. If $n=2^{a}$, then an obvious chain is given by $\left\{1,2,4, \ldots, 2^{a-1}, 2^{a}\right\}$. Any chain of length $a-1$ can have no element greater than $2^{a-1}$, hence the given chain is minimal.

2. If $n=2^{a}-2^{a-1}$, then $n=2^{a-1}$, and so $\ell_{L}^{-}(n)=a-1$ by the first case.

3. If instead $n=2^{a}-2^{a-2}$, then we can write $n=2^{a-1}+2^{a-2}$ and the result now follows from the proof for integers of the form $2^{a}+2^{b}$, which we give in case 5 .

4. If $n=2^{a}-2^{b}$ and $b \leq a-3$, then a Lucas addition-subtraction chain with length $a+1$ for $n$ is

$$
\left\{1,2,2^{2}, \ldots, 2^{a-b}, 2^{a-b}-1,2^{a-b+1}-2^{1}, \ldots, 2^{a}-2^{b}\right\} .
$$

From Volger's results, we know any chain for $n$ must have length at least $a+1$, and so the given chain is minimal.

5. If $n=2^{a}+2^{b}$, Volger's results give us that $\ell^{-}(n)=a+1$. So we need only note that there is a Lucas addition-subtraction chain of length $a+1$ for $n$ :

$$
\left\{1,2,2^{2}, \ldots, 2^{a-b}, 2^{a-b}+1,2^{a-b+1}+2^{1}, \ldots, 2^{a}+2^{b}\right\}
$$

That is, the chain consists of $a-b$ doublings, followed by a +1 step, and then $b$ doublings.

For integers with Hamming weight 3, we can also determine their minimal length exactly.

Proposition 2.1.2. If $n=2^{a}+2^{b}+2^{c}$, then $\ell_{L}^{-}(n)=a+2$.

Proof. As noted previously, it follows from Volger's results that $\ell^{-}(n)=a+2$ and so $\ell_{L}^{-}(n) \geq$ $a+2$. We can easily construct a chain of length $a+2$ for $n$. We first do $a-b$ doublings, followed by a +1 step. Then we do $b-c$ more doublings, followed by a +1 step and then $c$ doublings:

$$
\begin{aligned}
& \left\{1,2,2^{2}, \ldots, 2^{a-b}, 2^{a-b}+1,2^{a-b+1}+2^{1}, \ldots,\right. \\
& \left.2^{b-c}\left(2^{a-b}+1\right)=2^{a-c}+2^{b-c}, 2^{a-c}+2^{b-c}+1, \ldots, 2^{a}+2^{b}+2^{c}\right\} .
\end{aligned}
$$

This proves the desired result.

Proposition 2.1.3. If $n=2^{a}+2^{b}+2^{c}+2^{d}$, then $\ell_{L}^{-}(n)=a+2$ or $a+3$. 
Proof. If $\bar{s}_{2}(n)<3$ then $n$ is of the form $2^{a}$ or $2^{a} \pm 2^{b}$, and $\ell_{L}^{-}(n)=a+2$ by Proposition 2.1.1. For the rest of the proof, we can therefore assume $\bar{s}_{2}(n) \geq 3$ and so we have $\ell_{L}^{-}(n) \geq a+2$ by Volger's part 6). Just as we did in the proof of the previous proposition, we can easily create a chain of length $a+3$ for $n$. We first do $a-b$ doublings, followed by a +1 step, then $b-c$ more doublings, followed by a +1 step and then $c-d$ doublings. Finally, we do one last +1 step, and $d$ doublings.

$$
\begin{gathered}
\left\{1,2,2^{2}, \ldots, 2^{a-b}, 2^{a-b}+1,2^{a-b+1}+2^{1}, \ldots, 2^{b-c}\left(2^{a-b}+1\right)\right. \\
=2^{a-c}+2^{b-c}, 2^{a-c}+2^{b-c}+1, \ldots, 2^{c-d}\left(2^{a-c}+2^{b-c}+1\right), 2^{c-d}\left(2^{a-c}+2^{b-c}+1\right) \\
\left.=2^{a-d}+2^{b-d}+2^{c-d}, 2^{a-d}+2^{b-d}+2^{c-d}, \ldots, 2^{a}+2^{b}+2^{c}+2^{d}\right\} .
\end{gathered}
$$

This shows that $\ell_{L}^{-}(n) \leq a+3$.

To show we can have both $\ell_{L}^{-}(n)=a+2$ and $a+3$ for an integer $n$ with $h(n)=4$, we give some infinite families with these different minimal lengths.

Proposition 2.1.4. If $n=2^{a}+2^{b}+2^{c}+2^{d}$, with $a-b=c-d=j$ (meaning that $n=$ $\left.2^{a}+2^{a-j}+2^{c}+2^{c-j}\right)$, then $\ell_{L}^{-}(n)=a+2$.

Proof. Note that we can write $n$ as

$$
n=2^{c-j}\left(2^{a-c}+1\right)\left(2^{j}+1\right) .
$$

From Proposition 2.1.1, we know $\ell_{L}^{-}\left(2^{a-c}+1\right)=a-c+1$ and $\ell_{L}^{-}\left(2^{j}+1\right)=j+1$. By the factor method (Corollary 11 of [13]), we have

$$
\ell_{L}^{-}\left(\left(2^{a-c}+1\right)\left(2^{j}+1\right)\right) \leq \ell_{L}^{-}\left(\left(2^{a-c}+1\right)+\ell_{L}^{-}\left(\left(2^{j}+1\right)=a-c+j+2 .\right.\right.
$$

That is, we can construct a Lucas addition-subtraction chain for $\left(2^{a-c}+1\right)\left(2^{j}+1\right)$ of length $a-c+j+2$. If we augment that chain by $c-j$ doublings, then we will get a chain for $n$ of length $a-c+j+2+c-j=a+2$.

Proposition 2.1.5. If $n=2^{a}+2^{a-1}+2^{a-2}+2^{b}$ with $0<b<a-3$, then $\ell_{L}^{-}(n)=a+3$.

Proof. We know that

$$
n=2^{a}+2^{a-1}+2^{a-2}+2^{b}=2^{a+1}-2^{a-2}+2^{b}
$$

and from Volger's results

$$
\ell^{-}\left(2^{a+1}-2^{a-2}+2^{b}\right) \geq a+3
$$

so likewise $\ell_{L}^{-}(n) \geq a+3$. However, from Proposition 9 we see that $a+3$ is also an upper bound, and hence we have equality.

We conclude this section with two results we will need in the proof of our main theorem. The first gives another infinite family with $h(n)=4$, and $\ell^{-}(n)=a+2$. The second shows that any minimal chain for integers of Hamming weight 2 contains only elements of a certain form.

Proposition 2.1.6. If $n=2^{a}+7 \cdot 2^{d}$ with $a \geq d+3$, then $\ell_{L}^{-}(n)=a+2$. 
Proof. The proof will be done in two cases. We first investigate the case where $d=0$ or 1 , and then after treat the case $d>1$.

If $d \leq 1$, then

$$
n=2^{a}+7 \cdot 2^{d}=2^{a}+\left(2^{3}-1\right) \cdot 2^{d}=2^{a}+2^{d+3}-2^{d}=2^{d+3}\left(2^{a-d-3}+1\right)-2^{d}
$$

and a chain can be constructed easily as follows:

$$
\begin{gathered}
c=\left\{1, \cdots, 2^{a-d-3}, 2^{a-d-3}+1,2 \cdot\left(2^{a-d-3}+1\right), \cdots,\right. \\
\left.2^{d+3} \cdot\left(2^{a-d-3}+1\right), 2^{d+3}\left(2^{a-d-3}+1\right)-2^{d}\right\} .
\end{gathered}
$$

If instead $d>1$ then we prove $\ell_{L}^{-}(n)=a+2$ by induction on $(a, d)$. When $(a, d)=(5,2)$, then $n=2^{5}+7 \cdot 2^{2}=60$, and a corresponding minimal chain is $\{1,2,4,8,7,15,30,60\}$ and $\ell^{-}(n)=a+2$. Let us now suppose now that the result holds for $(k, d)$ (with $2 \leq d \leq k-3$ ), and we will prove that it also holds for all $(k+1, d)$ with $2 \leq d \leq k-2$. We have

$$
n=2^{k+1}+7 \cdot 2^{d}=2\left(2^{k}+7 \cdot 2^{d-1}\right) .
$$

We know that $\ell_{L}^{-}\left(2^{k}+7 \cdot 2^{d-1}\right)=k+2$ by the induction hypothesis (as well as the case where $d-1 \leq 1$ which we treated previously). We simply add one doubling step to the chain for $2^{k}+7 \cdot 2^{d-1}$ to get a chain for $n=2^{k+1}+7 \cdot 2^{d}$ of length $k+3$, and the theorem holds.

We conclude this section with an important lemma we will need in the proof of our main result.

Lemma 2.2. The only elements in a minimal chain for $2^{a}+2^{b}$ are of the form $2^{i}$ with $i \leq a-b$, or $2^{a-b-1}+1$, or $2^{k}\left(2^{a-b}+1\right)$ where $k \leq b$.

Proof. Let us consider the chains

$$
c_{1}=\left\{1,2, \cdots, 2^{a-b}, 2^{a-b}+1,2\left(2^{a-b}+1\right), \cdots, 2^{b}\left(2^{a-b}+1\right)=n\right\}
$$

and

$$
c_{2}=\left\{1,2,, \cdots, 2^{a-b-1}, 2^{a-b-1}+1,2^{a-b}+1,2\left(2^{a-b}+1\right), \cdots, 2^{b}\left(2^{a-b}+1\right)=n\right\}
$$

which are minimal Lucas addition-subtraction chains for $n$. We will prove that they are the only possible such chains for $n$. For that, we assume we have some other minimal chain for $n$ and examine the first non-doubling step:

$$
c^{\prime}=\left\{1,2,, \cdots, 2^{\alpha}, c_{\alpha+1}^{\prime}, \cdots, n\right\} .
$$

We note the possible values for $c_{\alpha+1}^{\prime}$ are $2^{\alpha}+1$ or $2^{\alpha}-2^{\beta}$ or $2^{\alpha}+2^{\beta}$ and the total number of steps left (after $c_{\alpha+1}^{\prime}$ ) to reach $n$ is

$$
r=a+1-(\alpha+1)=a-\alpha .
$$

We will investigate the three cases and show they lead to the desired result. 
Case I: $c_{\alpha+1}^{\prime}=2^{\alpha}-2^{\beta}$ and thus the maximal value that we can reach from $c_{\alpha+1}$ is $2^{r}\left(c_{\alpha+1}\right)=$ $2^{a-\alpha}\left(2^{\alpha}-2^{\beta}\right)=2^{a}-2^{a-\alpha+\beta}<n$, which shows that this case is not possible.

Case II: $c_{\alpha+1}^{\prime}=2^{\alpha}+2^{\beta}$, with $\beta \geq 1$. This means that $2^{\alpha}-2^{\beta}$ belongs to the chain, and is consequently a power of 2 . The only possibility is $\beta=\alpha-1$.

So the chain $c^{\prime}$ is of the form

$$
c^{\prime}=\left\{1,2, \cdots, 2^{\alpha}, 2^{\alpha}+2^{\alpha-1}, \cdots, n\right\}
$$

Let us assume that there is a second non-doubling step. The chain $c^{\prime}$ will now look like:

$$
c=\{1,2,, \cdots, 2^{\alpha}, \underbrace{2^{\alpha}+2^{\alpha-1}}_{\text {non-doubling } 1}, \cdots, 2^{\beta}\left(2^{\alpha}+2^{\alpha-1}\right), \underbrace{c_{\alpha+\beta+2}^{\prime}}_{\text {non-doubling } 2}, \overbrace{\cdots, n}^{a-\alpha-\beta-1 \text { steps }}\}
$$

and

$$
c_{\alpha+\beta+2}^{\prime}= \begin{cases}2^{\beta}\left(2^{\alpha}+2^{\alpha-1}\right)+1, & \\ 2^{\beta}\left(2^{\alpha}+2^{\alpha-1}\right)+2^{\gamma}, & \text { with } 2^{\beta}\left(2^{\alpha}+2^{\alpha-1}\right)-2^{\gamma} \in c^{\prime} \text { and } \gamma \leq \alpha \\ 2^{\beta}\left(2^{\alpha}+2^{\alpha-1}\right)-2^{\gamma}, & \text { and } \gamma \leq \alpha \\ 2^{\beta}\left(2^{\alpha}+2^{\alpha-1}\right)+2^{\gamma}\left(2^{\alpha}+2^{\alpha-1}\right), & \text { with }\left(2^{\alpha}+2^{\alpha-1}\right)\left(2^{\beta}-2^{\gamma}\right) \in c^{\prime} \text { and } \gamma \leq \beta \\ 2^{\beta}\left(2^{\alpha}+2^{\alpha-1}\right)-2^{\gamma}\left(2^{\alpha}+2^{\alpha-1}\right), & \text { and } \gamma \leq \beta .\end{cases}
$$

Examining each of these 5 cases shows they are not possible because even if the remaining steps are all doublings, then the maximum value in the chain that will be reached is of the form

$$
2^{a-\alpha-\beta-1}\left(c_{\alpha+\beta+2}\right)=2^{a-1}+\cdots<2^{a}<n .
$$

So all the remaining steps must be doublings, and we have

$$
n=2^{r}\left(c_{\alpha+1}\right)=2^{a-\alpha}\left(2^{\alpha}+2^{\alpha-1}\right)=2^{a}+2^{a-1} .
$$

This is impossible if $a>b+1$, and so we must have $b=a-1$. We conclude that every element of the chain is one of the desired forms.

Case III: $c_{\alpha+1}^{\prime}=2^{\alpha}+1$. If $\alpha=a-b$ then the remaining steps must all be doublings, else the chain's maximum value will be less than $n$. If $\alpha=a-b-1$, then $c_{\alpha+1}^{\prime}=2^{a-b-1}+1, c_{\alpha+2}^{\prime}=$ $c_{\alpha}^{\prime}+c_{\alpha+1}^{\prime}=2^{a-b}+1$, followed by $b$ doublings.

If $\alpha>a-b$, then the maximum value that the chain can reach is $2^{a-\alpha}\left(2^{\alpha}+1\right)=2^{a}+2^{a-\alpha}<$ $n$.

If $\alpha<a-b-1$, then the maximum value that the chain can reach is $2^{a-\alpha}\left(2^{\alpha}+1\right)=$ $2^{a}+2^{a-\alpha}>n$. However, this means that the minimal chain for $n$ must contain a back step (a step involving subtraction). Using a result from [14], we have that

$$
n \leq 2^{d-1} F_{f+3},
$$

with $d$ and $f$ being respectively the number of doubling and the number of back steps, and $\left(F_{n}\right)$ is the $n$-th Fibonacci number. Having $f \geq 1$ implies that $n \leq 2^{a}$ which leads to a contradiction. 


\section{The main result}

Our main result compares $\ell_{L}^{-}(n / 2)$ and $\ell_{L}^{-}(n)$, for even integers with low Hamming weight.

Theorem 3.1. If the Hamming weight of an even integer $n$ is $\leq 4$, then

$$
\ell_{L}^{-}(n / 2)+1=\ell_{L}^{-}(n) .
$$

Proof. Let $n$ be an even integer. When the Hamming weight of $n$ is three or less, the results easily follow from Theorem 6 and the fact that $s_{2}(n)=s_{2}(n / 2)$. For example, if $n=2^{a}+2^{b}$, then $\ell_{L}^{-}(n)=a+1=\ell_{L}^{-}\left(2^{a-1}+2^{b-1}\right)+1=\ell_{L}^{-}(n / 2)+1$. Similarly, if the minimal weight is three or less it is easy to see the result holds. If $\bar{s}_{2}(n)=2$, we use Proposition 2.1.1. When $\bar{s}_{2}(n)=3$, then $n=2^{x} \pm 2^{y} \pm 2^{z}$ and $\ell_{L}^{-}\left(2^{x} \pm 2^{y} \pm 2^{z}\right)=x+2$, except when $y=x-3, z=\{x-4, x-5\}$ and then $\ell_{L}^{-}\left(2^{x}-2^{x-3}-2^{z}\right)=x+1$ (see $\left.[13,14]\right)$. In either case, $\ell_{L}^{-}(n)=\ell_{L}^{-}(n / 2)+1$.

We now turn to the more difficult case when $s_{2}(n)=4$, i.e., $n=2^{a}+2^{b}+2^{c}+2^{d}$. From above, we can also take the minimal weight $\bar{s}_{2}(n)$ to be at least 4. By Proposition 2.1.3, we know that $\ell_{L}^{-}(n)=a+2$ or $a+3$. Assume first that the minimal chain is of length $a+3$. Then $n / 2=2^{a-1}+2^{b-1}+2^{c-1}+2^{d-1}$, and so $\ell_{L}^{-}(n / 2)=a+1$ or $a+2$. If it were $a+1$, then we could obviously create a chain for $n$ of length $a+2$, by simply appending $n$. This is a contradiction, and so $\ell_{L}^{-}(n / 2)=a+2$ and equation (2) holds.

For the remainder of the proof we therefore assume $\ell_{L}^{-}(n)=a+2$. Let

$$
C=\left\{c_{0}, c_{1}, c_{2}, \cdots, c_{a+1}, c_{a+2}\right\}
$$

be a minimal Lucas addition-subtraction chain for $n$. We have four possible relations between $c_{a+1}$ and $c_{a+2}$ (see Definition 4). If $c_{a+2}=c_{a+1}+1$, then $c_{a+1}$ also has Hamming weight at least 4 , and so $\ell_{L}^{-}\left(c_{a+1}\right) \geq a+2$, a contradiction. If $c_{a+2}=2 c_{a+1}$, then $\left\{c_{0}, c_{1}, c_{2}, \cdots, c_{a+1}\right\}$ is a chain for $n / 2$ and clearly $\ell_{L}^{-}(n / 2)+1=\ell_{L}^{-}(n)$.

The remaining two cases are when $c_{a+2}=c_{a+1} \pm c_{f}$, for some $c_{f}$ in the chain. For the first case, $c_{a+2}=c_{a+1}-c_{f}$, then $c_{a+1}>n$. It must be that $c_{a+1}$ has Hamming weight less than three, since otherwise $\ell_{L}^{-}\left(c_{a+1}\right) \geq a+2$, which contradicts that we already have a chain for $c_{a+1}$ of length $a+1$. If the Hamming weight of $c_{a+1}$ were 1 , then it follows $c_{a+1}=2^{a+1}$ and $c_{l}=2^{l}$ for all $l=1, \cdots, a+1$. This means that $n$ is of the form $2^{a+1}-2^{k}$, for some $k$, and so $\bar{s}_{2}(n)=2$, a case we already dealt with. The remaining possibility in this case is that the Hamming weight of $c_{a+1}$ is 2 , so $c_{a+1}=2^{a}+2^{j}$, for some $j>b$. Note that we know $c_{a+1} \neq 2^{a+1}+2^{j}$, as with $a+1$ steps, the maximum value in a chain is $2^{a+1}$. By Lemma 2.2, the only steps in a chain for integers of the form $2^{a}+2^{j}$ are of the form $2^{i}, 2^{i}\left(2^{a-j}+1\right)$, or $2^{a-j-1}+1$. We get that $n$ must be of the form:

$$
n=2^{a}+2^{j}-2^{i} \text { or } n=2^{a}+2^{j}-2^{i}\left(2^{a-j}+1\right) \text { or } n=2^{a}+2^{j}-2^{a-j-1}-1 .
$$

Since $c_{a+1} \geq 2^{a}$, we know that any minimal chain for it must have length at least $a+1$. But it is easy to see that no matter which of the first two forms $n$ has, there are chains of length $a+1$ for $n / 2$, showing that $\ell_{L}^{-}(n / 2)=a+1$. More concretely, for $n=2^{a}+2^{j}-2^{i}$, perform $a-j$ doublings, do one +1 step, then $j-1$ more doublings, followed by a back step of subtracting $2^{i-1}$. 
Similarly, for $n=2^{a}+2^{j}-2^{i}\left(2^{a-j}+1\right)$, begin with $a-j$ doublings, then do a +1 step, then $j-1$ doublings and finally a back step of subtracting $2^{i-1}\left(2^{a-j}+1\right)$. The case $n=2^{a}+2^{j}-2^{a-j-1}-1$ is not possible since $n$ is even.

The final case left to consider is $c_{a+2}=c_{a+1}+c_{f}$, which implies that $\ell_{L}^{-}\left(c_{a+1}\right) \leq a+1$. We examine several subcases depending on whether $\ell_{L}^{-}\left(c_{a+1}\right)<a, \ell_{L}^{-}\left(c_{a+1}\right)=a$, or $\ell_{L}^{-}\left(c_{a+1}\right)=a+1$.

1. $\ell_{L}^{-}\left(c_{a+1}\right)<a$, meaning that $c_{a+1} \leq 2^{a-1}$. From this we can deduce that $c_{f}=2^{a}$, or $2^{a-1}+2^{\eta}$, since $c_{f} \geq 2^{a-1}+2^{b}+2^{c}+2^{d}$ and $\ell_{L}^{-}\left(c_{f}\right) \leq a$. If $c_{f}=2^{a-1}+2^{\eta}$, then $c_{a+1}=n-c_{f}=2^{a-1}+2^{b}+2^{c}+2^{d}-2^{\eta} \leq 2^{a-1}$. In fact, the inequality must be strict as we cannot have $2^{b}+2^{c}+2^{d}=2^{\eta}$. However, then we can conclude that $\ell_{L}^{-}\left(c_{a+1}\right) \geq$ $\ell^{-}\left(c_{a+1}\right) \geq\left\lfloor\log \left(c_{a+1}\right)\right\rfloor+2=a$ which contradicts $\ell_{L}^{-}\left(c_{a+1}\right)<a$.

If instead $c_{f}=2^{a}$, then $c_{a+1}=2^{b}+2^{c}+2^{d}$. The only possible chain is

$$
C=\left\{1,2,4, \cdots, c_{f}=c_{a}=2^{a}, c_{a+1}, c_{a+2}\right\} .
$$

Then (for some $i$ and $j$ ) we have $c_{a+1}=2^{i} \pm 2^{j}=2^{b}+2^{c}+2^{d}$ which is only possible when $i-j=3$ and $b=c+1=d+2$. We can then write $n$, as well as $n / 2$, in the form $2^{a}+7 \cdot 2^{d}$. By Lemma 2.1.6, the desired result holds.

2. If $c_{a+1} \leq n / 2$ and $\ell_{L}^{-}\left(c_{a+1}\right)=\{a, a+1\}$, then necessarily $c_{f} \geq n / 2$ which means that $a \geq \ell_{L}^{-}\left(c_{f}\right) \geq \ell^{-}\left(c_{f}\right) \geq\left\lceil\log \left(c_{f}\right)\right\rceil \geq a$. But then $c_{f}$ is either $2^{a}$ or $2^{a-1}+2^{\alpha}$ with $\alpha>b-1$. If $c_{f}=2^{a}$, then $c_{a+1}=2^{i} \pm 2^{j}$ with $a \geq i>j \geq 0$ since $c_{a+1}$ is obtained from the minimal chain for $c_{f}$. However, this implies $2^{b}+2^{c}+2^{d}=2^{i} \pm 2^{j}$, which leads to the desired result as shown above in the last case.

If $c_{f}=2^{a-1}+2^{\alpha}$, then the only possibilities for $c_{s}=c_{f}-c_{a+1}$ are $2^{i}$ or $2^{i}\left(2^{a-1-\alpha}+1\right)$ or $2^{a-1-\alpha}+1$ and so $n=c_{a+1}+c_{f}=2 c_{f}-c_{s}$ has the following possibilities $2^{a}+2^{\alpha+1}-2^{k}$ or $2^{a}+2^{\alpha+1}+2^{k}\left(2^{a-\alpha-1}+1\right)$ or $2^{a}+2^{\alpha+1}-2^{a-1-\alpha}-1$. If $n=2^{a}+2^{\alpha+1}-2^{k}$, then as seen above it must be that $n=2^{a}+7 \cdot 2^{d}$. If $n=2^{a}+2^{\alpha+1}-2^{k}\left(2^{a-\alpha-1}+1\right)=$ $2^{\alpha+1}\left(2^{a-\alpha-1}+1\right)-2^{k}\left(2^{a-\alpha-1}+1\right)$, then

$$
\begin{gathered}
c=\left\{1,2, \cdots, 2^{a-\alpha-1}, 2^{a-\alpha-1}+1,2\left(2^{a-\alpha-1}+1, \cdots, 2^{k}\left(2^{a-\alpha-1}+1\right), \cdots,\right.\right. \\
\left.2^{\alpha+1}\left(2^{a-\alpha-1}+1\right), n=2^{\alpha+1}\left(2^{a-\alpha-1}+1\right)-2^{k}\left(2^{a-\alpha-1}+1\right)\right\},
\end{gathered}
$$

which is a chain for $n$ of length $a+2$. We can similarly construct a chain for $n / 2$ of length $a+1$ (we just replace $\alpha$ by $\alpha-1$ and $k$ by $k-1$ in the expression of $n$ to get $n / 2$ ). The case $n=2^{a}+2^{\alpha+1}-2^{a-1-\alpha}-1$ is not possible since $n$ is even.

3. $\ell_{L}^{-}\left(c_{a+1}\right)=a$ and $c_{a+1}>n / 2$.

As $c_{a+1}>n / 2$, then it follows that $c_{a+1}=2^{a}$ or $2^{a-1}+2^{\alpha}$, with $\alpha<a-1$; otherwise $c_{a+1}>n$ which contradicts our assumption that $n$ isn't obtained from a subtraction step. Note also that $\alpha \geq b$, otherwise $c_{a+1}<n / 2$. We suppose first $c_{a+1}=2^{a}$, and so $c_{f}=$ $2^{b}+2^{c}+2^{d}$ and the chain for $n$ must be

$$
C=\{c_{0}, c_{1}, c_{2}, \cdots, \underbrace{2^{b}+2^{c}+2^{d}}_{c_{f}}, \cdots, \underbrace{2^{a}}_{c_{a+1}}, \underbrace{n}_{c_{a+2}}\} .
$$


We note that $2^{a}$ has been reached with $a+1$ steps, meaning the only non doubling step involves $c_{f}$. But then $c_{f}=2^{b}+2^{c}+2^{d}$ can only be obtained from the sum or the difference of two powers of 2 . We have already observed this is only possible if $b=c+1=d+2$, meaning $n=2^{a}+2^{d+2}+2^{d+1}+2^{d}=2^{a}+7 \cdot 2^{d}$. The result now follows from an appeal to Lemma 2.1.6.

The remaining possibility in this subcase is if $c_{a+1}=2^{a-1}+2^{\alpha}$, which makes $c_{f}=2^{a-1}+$ $2^{b}+2^{c}+2^{d}-2^{\alpha}$. If $\alpha$ is equal to one of $b, c$, or $d$, then $c_{f}$ has Hamming weight 3. By Theorem 2.1, $\ell_{L}^{-}\left(c_{f}\right)=(a-1)+2=a+1$. This is a contradiction, since $c_{f}$ appeared in the chain $C$ before $a+1$ steps. On the other hand, if $\alpha \neq b$ and $\alpha \neq c$ and $\alpha \neq d$ then it's not to hard to check that $s^{-}\left(c_{f}\right) \geq 3$ (the assumption $2^{a-1}+2^{b}+2^{c}-2^{\alpha}=2^{i} \pm 2^{j}$ leads to a contradiction). Then using Volger's inequality

$$
\ell_{L}^{-}\left(c_{f}\right) \geq \ell^{-}\left(c_{f}\right) \geq\left\lfloor\log \left(c_{f}\right)\right\rfloor+2=(a-1)+2=a+1 .
$$

This is again a contradiction.

4. $\ell_{L}^{-}\left(c_{a+1}\right)=a+1$, and $c_{a+1}>n / 2$. Since $c_{a+1}>n / 2$, we must have that either $c_{a+1}=$ $2^{a-1}+2^{\alpha}+2^{\beta}$ or $c_{a+1}=2^{a}+2^{\alpha}$ (with $\alpha, \beta<a$ ). Here, we have omitted the cases $c_{a+1}=2^{a}-2^{\alpha}$ and $c_{a+1}=2^{a-1}+2^{\alpha}-2^{\beta}$ with $\alpha>b$ to keep the condition $c_{a+1}>n / 2$. If $c_{a+1}=2^{a}-2^{\alpha}$, then $n=2^{a}-2^{\alpha}+2^{i}$ or $n=2^{a}-2^{\alpha}+2^{i}\left(2^{a-\alpha}-1\right)=2^{i}\left(2^{\alpha-i}+\right.$ 1) $\left(2^{a-\alpha}-1\right)$. The case $n=2^{a}-2^{\alpha}+2^{i}$ is not possible since $\bar{s}_{2}(n)=4$. The case $n=2^{i}\left(2^{\alpha-i}+1\right)\left(2^{a-\alpha}-1\right)$ is possible and we obtain the appropriate chain using the factor method. If $c_{a+1}=2^{a-1}+2^{\alpha}-2^{\beta}$, then $c_{f}=2^{a-1}+2^{b}+2^{c}+2^{d}-2^{\alpha}+2^{\beta}$. This case will be simultaneously studied with $c_{a+1}=2^{a-1}+2^{\alpha}+2^{\beta}$ leading to $c_{f}=$ $2^{a-1}+2^{b}+2^{c}+2^{d}-2^{\alpha}-2^{\beta}$.

Supposing first that $c_{a+1}=2^{a}+2^{\alpha}$, we know by Lemma 2.2 that $c_{f}$ is of the form $2^{i}$ or $2^{j}\left(2^{a-\alpha}+1\right)$ or $2^{a-\alpha-1}+1$, for some $i$ or $j$. If $c_{f}=2^{i}$, then $n=c_{a+1}+c_{f}=2^{a}+2^{\alpha}+2^{i}$, which would contradict $n$ having Hamming weight 4. If $c_{f}=2^{a-\alpha-1}+1$ then $n=$ $2^{a}+2^{\alpha}+2^{a-\alpha-1}+1$, which contradicts $n$ being even. Alternatively, if $c_{f}=2^{j}\left(2^{a-\alpha}+1\right)$ then

$$
n=2^{a}+2^{b}+2^{c}+2^{d}=2^{a}+2^{\alpha}+2^{a-\alpha+j}+2^{j} .
$$

Checking the possible combinations, we must either have $(b=\alpha$ and $c=a-\alpha+j$ and $d=j$ ), or alternatively $(b=a-\alpha+j, c=\alpha$, and $d=j)$. In both situations, we see that this implies $a-b=c-d$. By Proposition 2.1.4, $\ell_{L}^{-}(n)=a+2$. But then Proposition 2.1.4 also applies to $n / 2$, which proves the result we want.

The final subcase to investigate is when $c_{a+1}=2^{a-1}+2^{\alpha} \pm 2^{\beta}$. It follows that $\left\{c_{0}, c_{1}, c_{2}, \cdots\right.$, $\left.c_{a+1}\right\}$ is a minimal Lucas addition-subtraction chain for $c_{a+1}$. Since $c_{a+2}=c_{a+1}+c_{f}$, then

$$
c_{f}=2^{a-1}+2^{b}+2^{c}+2^{d}-2^{\alpha} \pm 2^{\beta} .
$$

We investigate $c_{f}$ depending on the possibilities for $\alpha$ and $\beta$. First, one can notice that $c_{f}>2^{a-2}$ and $\ell_{L}^{-}\left(c_{f}\right) \leq a$, and so therefore $\ell_{L}^{-}\left(c_{f}\right)$ is either $a, a-1$ or $a-2$, implying 
$\bar{s}_{2}\left(c_{f}\right) \leq 3$. On the other hand, it is easy to check that:

$$
\bar{s}_{2}\left(c_{f}\right)= \begin{cases}2 & \text { when } \alpha=b \text { and } \beta=c \text { or } \beta=d, \\ 2 & \text { when } \alpha=b+1 \text { and } 2^{c}+2^{d}+2^{\beta}=2^{b}-2^{I}, \\ 3 & \text { when } \alpha=b \text { and } \beta=\{c \pm 1, d \pm 1\} \\ 3 & \text { when } \alpha=b \text { and } 2^{c}+2^{d}+2^{\beta}=2^{I}-2^{J}, \\ 3 & \text { when } \alpha=b+1 \text { and } 2^{c}+2^{d}+2^{\beta}=2^{b-1}-2^{I}, \\ 3 & \text { when } \alpha>b+1 \text { and } 2^{b}+2^{c}+2^{d}+2^{\beta}=2^{I}-2^{J} \text { and } I=\alpha-1, \\ \geq 4 & \text { in all others general cases and they aren't of interest here. }\end{cases}
$$

when $c_{f}=2^{a-1}+2^{b}+2^{c}+2^{d}-2^{\alpha} \pm 2^{\beta}$.

We conclude by examining each of the possibilities for $c_{f}$. The possibilities for $\bar{s}_{2}\left(c_{f}\right)=2$ are:

- $c_{f}=2^{a-1}+2^{c}$,

- $c_{f}=2^{a-1}+2^{d}$,

- $c_{f}=2^{a-1}-2^{I}$ with $2^{c}+2^{d}+2^{\beta}=2^{b}-2^{I}(I=b-3)$.

- $c_{f}=2^{a-1}-2^{J}$ with $2^{b}+2^{c}+2^{d}+2^{\beta}=2^{I}-2^{J}$ and $I=\alpha=a-2$.

The case when $c_{f}=2^{a-1}+2^{d}$, meaning that $c_{a+1}=2^{a-1}+2^{b}+2^{c}$, is not possible since we cannot obtain $c_{a+1}$ from a minimal Lucas addition -subtraction chain for $c_{f}$ due to the fact that $b>c>d$. Similar logic holds for when $c_{f}=2^{a-1}+2^{c}$. If $c_{f}=2^{a-1}-2^{I}$, then $I=b-3$ and $\alpha=a-2, \beta=\{b+1, b-1, b-2, b-3\}$. Then $n$ will be $2^{a}+2^{a-1}+2^{b+1}-2^{b-3}-2^{\beta}$ and it leads to contradictions with the fact that $n=2^{a}+2^{b}+2^{c}+2^{d}$ for all the values of $\beta$ except $\beta=b+1$ which leads to $n=2^{a}+7 \cdot 2^{a-4}$ for which the proof holds. If $c_{f}=2^{a-1}-2^{J}$ then $I=a-2, J=a-6, \beta=\{b+1, b-1, b-2, b-3\}$ and it leads to $n \in\left\{71 \cdot 2^{k+6}, 39 \cdot 2^{k+5}, 75.2^{k+6}, 77 \cdot 2^{k+6}\right\}$ and the proof holds for all these possibilities of $n$. For example, one can easily see that $\ell_{L}^{-}\left(71 \cdot 2^{k+6}\right)=\ell_{L}^{-}(71)+k+6$, and the main result clearly holds for numbers of this form.

The possibilities for $\bar{s}_{2}\left(c_{f}\right)=3$ are:

- $c_{f}=2^{a-1}-2^{c}+2^{d}, c_{f}=2^{a-1}+2^{c}-2^{d}$,

- $c_{f}=2^{a-1}+2^{c-1}+2^{d}, c_{f}=2^{a-1}+2^{c}+2^{d-1}$,

- $c_{f}=2^{a-1}+2^{I}-2^{J}$ when $2^{c}+2^{d}+2^{\beta}=2^{I}-2^{J}$,

- $c_{f}=2^{a-1}-2^{b}+2^{c}, c_{f}=2^{a-1}-2^{b}+2^{d}$,

- $c_{f}=2^{a-1}-2^{b-1}-2^{I}$ when $2^{c}+2^{d}+2^{\beta}=2^{I}-2^{J}$ with $I=b-1$,

- $c_{f}=2^{a-1}-2^{I}-2^{J}$ when $2^{b}+2^{c}+2^{d}+2^{\beta}=2^{I}-2^{J}$ with $I=\alpha-1$,

- $c_{f}=2^{a-2}+2^{I}-2^{J}$ when $2^{b}+2^{c}+2^{d}+2^{\beta}=2^{I}-2^{J}$ with $I \neq a-2$. 
All these cases lead to $\ell_{L}^{-}\left(c_{f}\right)=a+1>a$ which is impossible, except for $c_{f}=2^{a-1}-$ $2^{b-1}-2^{I}$ or $c_{f}=2^{a-1}-2^{I}-2^{J}$ or $c_{f}=2^{a-2}+2^{I}-2^{J}$ for which $\ell_{L}^{-}\left(c_{f}\right)=a$. If $c_{f}=2^{a-1}-2^{b-1}-2^{I}$ or $c_{f}=2^{a-1}-2^{I}-2^{J}$, then $b-1=a-1-3, I=\{a-1-4, a-1-5\}$ or $I=a-1-3, J=\{a-1-4, a-1-5\}$. One can easily see that these two cases are also impossible since $I=b-1$ in the first case and $I-J>3$ in the second case. If $c_{f}=2^{a-2}+2^{I}-2^{J}$, then $c_{a+1}=2^{a-1}+2^{a-2}-2^{\beta}$ since $\alpha=a-2$ and $I=\max b, \beta+1>\beta \geq J$. If $\beta=J$, then $n=2^{a}+2^{I}-2^{I-3}=2^{a}+7 \cdot 2^{I-3}$ and the proof holds. If $\beta>J$, then $n=\left\{2^{a}+2^{I-1}+2^{J}, 2^{a}+2^{I-2}+2^{J}\right\}$ and both cases are impossible.

Having exhausted all possibilities, we see that in every case we have demonstrated that if $h(n) \leq 4$, then $\ell_{L}^{-}(2 n)=\ell_{L}^{-}(n)+1$.

As an immediate corollary, we have the following:

Corollary 3.1.1. If $s_{2}(n) \leq 4$, then $\ell_{L}^{-}\left(2^{k} n\right)=\ell_{L}^{-}(n)+k$.

\section{Conclusion}

We can ask a question related to Hansen numbers [6]: Is there any integer $n$ such that $\ell_{L}^{-}(2 n)<$ $\ell_{L}^{-}(n)$ ? This conjecture is known to be true for addition chains. Namely, for $n=375404703$, $\ell(n)=35>34=\ell(2 n)$, see [2]. The analogous question remains open for addition-subtraction chains, and Lucas addition chains. Our paper has proven that there is no number verifying the conjecture with Hamming weight $\leq 4$. In fact, we also proved that $\left|\ell^{-}(2 n)-\ell^{-}(n)\right| \leq 1$ for all Hamming weight $\leq 4$. One can investigate if $\ell^{-}(2 n)=\ell^{-}(n)=a+2$ for some integers $n$ of Hamming weight 4 .

The techniques used in the proofs of this paper could also be investigated further in order to design a polynomial-time algorithm for computing fast exponentiations. We leave this for future work.

\section{Acknowledgements}

The authors would like to acknowledge the precious support of Maurice Mignotte. This work was finalized during a research visit of one of the authors at the Max-Planck Institute for Mathematik in Bonn MPIM.

\section{References}

[1] M. Ciet, M. Joye, K. Lauter,\& Montgomery, P. L. (2006). Trading inversions for multiplications in elliptic curve cryptography, Des. Codes Cryptogr., 39, 189-206. 
[2] Cliff, N. (2011) Shortest addition chains. Available online:

http://wwwhomes.uni-bielefeld.de/achim/addition_chain.html.

[3] Dimitrov, V., Imbert, L. \& Mishra, P. K. (2005). Efficient and secure elliptic curve point multiplication using double-base chains, in Advances in Cryptology - ASIACRYPT 2005, Lect. Notes in Comp. Sci., 3788, Springer-Verlag, 59-78.

[4] Downey, P., Leong, B. \& Sethi, R. (1981). Computing sequences with addition chains, SIAM J. Comput., 10, 638-646.

[5] Gordon, D.M. (1998). A survey of fast exponentiation methods, Journal of Algorithms, 27, 129-146.

[6] Knuth, D. (1969) The Art of Computer Programming, 2nd ed., Addison-Wesley.

[7] Koyama, K. \& Tsuruoka, Y. (1993). Speeding elliptic cryptosystems using a signed binary window method, in Advances in Cryptology - CRYPTO 1993, Lect. Notes in Comput. Sci. 740, Springer-Verlag, 345-357.

[8] Le, D. (2011). Fast quadrupling of a point in elliptic curve cryptography, preprint, 2011, Available online: https://eprint.iacr.org/2011/039.

[9] Montgomery, P. (1992). Evaluating recurrences of form $X_{m+n}=f\left(X_{m}, X_{n}, X_{m-n}\right)$ via Lucas chains, preprint, 1992, Available online: ftp://ftp.cwi.nl/pub/ pmontgom/Lucas.ps.gz.

[10] Morain, F. \& Olivos, J. (1990). Speeding up the computation on an elliptic curve using addition-subtaction chains, RAIRO Theor. Inform. Appl., 24, 531-543.

[11] Sakai, Y. \& Sakurai, K. (2001). Efficient scalar multiplication on elliptic curve with direct computations of several doublings, IEICE Transactions on Fundamentals of Electronics, Communications and Computer Sciences, 84, 120-129.

[12] Takagi, T., Reis, D., Yen, S. \& Wu, B. (2006). Radix-r non-adjacent form and its application to pairing-based cryptosystem, IEICE Transactions on Fundamentals of Electronics, Communications and Computer Sciences, 89, 115-123.

[13] Tall, A. (2013). A generalization of Lucas addition chains, Bull. Math. Soc. Sci. Math. Roumanie 55, 1. Available online: https://eprint.iacr.org/2011/378.pdf.

[14] Volger, H. (1985). Some results on addition/subtraction chains, Inform. Process. Lett., 20, $155-160$.

[15] Wang, C., Chang, C. \& Lin, C. (1999). A method for computing Lucas sequences, Comput. Math. Appl., 38, 187-196.

[16] Yacobi, Y. (1991). Exponentiating faster with addition chains, In: Advances in Cryptology - EUROCRYPT 1990 Lect. Notes in Comput. Sci., Vol. 473, Springer-Verlag, 222-229. 